\title{
COVID-19 related mental health burdens: Impact of educational level and relationship status among low income earners of Western Uganda
}

Lemuel Ann Monima ( $\sim$ annexdot5@gmail.com )

Kampala International University - Western Campus https://orcid.org/0000-0002-6998-1439

Ibe Michael Usman

Kampala International University - Western Campus

Kenneth Iceland Kasozi

The University of Edinburgh

Eric . Osamudiamwen Aigbogun, Jr

Kampala International University - Western Campus

Victor Archibong Bassey

Kampala International University - Western Campus

Josiah Ifie

Kampala International University - Western Campus

Robinson Ssebuufu

Kampala International University - Western Campus

Adam Moyosore Afodun

Kampala International University - Western Campus

Swase Dominic Terkimbi

Kampala International University - Western Campus Ngala Elvis Mbiydzenyuy

The Copperbelt University School of Medicine

Azeez Adeoye

Kampala International University - Western Campus Joshua Ojodale Aruwa

Kampala International University - Western Campus

Odoma Saidi

Kampala International University - Western Campus

Fred Ssempijja

Kampala International University - Western Campus

John Tabakwot Ayuba

Kampala International University - Western Campus

Hope Onohuean 
Kampala International University - Western Campus

\section{Stellamaris Kembabazi}

Kampala International University - Western Campus

\section{Theophilus Pius}

Kampala International University - Western Campus

\section{Viola Nankya}

Kampala International University - Western Campus

\section{Kevin Matama}

Kampala International University - Western Campus

\section{Rachael Henry}

Ahmadu Bello University

\section{Helen Yusuf}

Ahmadu Bello University

\section{Susan Christina Welburn}

The University of Edinburgh Edinburgh Medical School

\section{Research Article}

Keywords: Mental healthcare, awareness, relationship status, educational level, COVID-19, Low-income earners, Western Uganda

Posted Date: August 20th, 2020

DOI: https://doi.org/10.21203/rs.3.rs-61564/v1

License: (c) (i) This work is licensed under a Creative Commons Attribution 4.0 International License. Read Full License 


\section{COVID-19 related mental health burdens: Impact of educational level and 2 relationship status among low income earners of Western Uganda}

\section{Authors list}

Ann Monima Lemuel ${ }^{*}$, Ibe Michael Usman ${ }^{1 *}$, Kenneth Iceland Kasozi ${ }^{2 *}$, Eric Osamudiamwen Aigbogun, Jr. ${ }^{1}$, Victor Archibong Bassey $^{1}$, Ifie Josiah ${ }^{1}$, Robinson Ssebuufu ${ }^{3}$, Adam Moyosore Afodun ${ }^{1}$, Swase Dominic Terkimbi ${ }^{1}$, Ngala Elvis Mbiydzenyuy ${ }^{4}$, Azeez Adeoye $^{1}$, Joshua Ojodale Aruwa ${ }^{1}$, Odoma Saidi ${ }^{1}$, Fred Ssempijja ${ }^{1}$, John Tabakwot Ayuba ${ }^{1}$, Hope Onohuean ${ }^{1}$, Stellamaris Kembabazi ${ }^{1}$, Theophilus Pius ${ }^{5}$, Viola Nankya ${ }^{6}$, Kevin Matama ${ }^{7}$, Rachael Henry ${ }^{8}$, Helen Yusuf ${ }^{8}$, and Susan Christina Welburn ${ }^{2,9^{*}}$

1. Faculty of Biomedicals Sciences, Kampala International University Western Campus, Bushenyi, Uganda

2. Infection Medicine, Deanery of Biomedical Sciences, College of Medicine and Veterinary Medicine, The University of Edinburgh, Edinburgh, United Kingdom

3. Faculty of Clinical Medicine and Dentistry, Kampala International University Teaching Hospital, Bushenyi, Uganda

4. Department of Basic Medical Science, School of Medicine, Copperbelt University, Ndola Zambia

5. Department of Medical Laboratory Science, Kampala International University, Uganda

6. School of Nursing, Kampala International University Teaching Hospital, Bushenyi, Uganda

7. School of Pharmacy, Kampala International University Western Campus, Kampala, Uganda

8. Department of Human Anatomy, College of Medicine and Health Science, Ahmadu Bello University, Zaria, Nigeria

9. Zhejiang University-University of Edinburgh Institute, Zhejiang University School of Medicine, International Campus, Zhejiang University, Haining, China

\section{*Corresponding Authors:}

21 Ann Lemue email: annexdot5@gmail.com

22 Ibe Michael Usman email: gopama13@gmail.com

23 Kenneth Iceland Kasozi email: kicelandy@gmail.com

24 Susan Christina Welburn email: sue.welburn@ed.ac.uk 


\section{Abstract}

Introduction: The imposed COVID-19 lockdown is taking a toll mental health, with low-income earners considered particularly affected. The study aimed to investigate level of education and relationship status on awareness of mental healthcare and explore how these factors influence mental health among low income earners in Western Uganda.

Methods: A cross sectional descriptive study was undertaken among low-income earners in South Western Uganda. Data were collected from two hundred and fifty-three (253) participants using a structured closed ended questionnaire. Anxiety, anger and depression were assessed using a modified generalized anxiety disorder (GAD-7), Spielberger's State-Trait Anger Expression Inventory-2 (STAXI-2) and Beck Depression Inventory (BDI) item tools respectively.

Result: Most respondents were single male individuals with secondary level. Respondents with tertiary level of education and those with partners had higher score $(71.7 \%$ and $70.28 \%$ respectively) for awareness on mental healthcare. Respondents with primary education level and individuals that were single had higher score (46.98\% and $43.72 \%$ respectively) for anxiety. Respondents with primary education level and those living with partners had higher mean score (56.44\% and 56.21\% respectively) for anger. Respondents with no formal education and those who had lost partners had higher mean score $(32.11 \%$ and $30.50 \%$ respectively) for depression. Individuals without formal educational and primary education were observed to have a negative correlation $\left(\mathrm{r}^{2}=47.4\right.$ and $6.4 \%$ respectively) with awareness on mental healthcare, and the no formal group had positive correlation with anger and depression $\left(\mathrm{r}^{2}=1.9\right.$ and $0.3 \%$ respectively), while other groups were negatively correlated $\left(r^{2}=10.1 \%\right)$. The group of single individuals had a negative correlation with awareness on mental healthcare, anger and depression $\left(r^{2}=1.9,0.8\right.$ and $0.3 \%$ respectively), and a positive correlation with anxiety $\left(\mathrm{r}^{2}=3.9 \%\right)$.

Conclusion: It is evident that education and relationship status influence awareness on mental healthcare and mental health state among low income earners in Western Uganda. Therefore, we advocate for new approaches in mental healthcare awareness programs equipped with robust strategy, which will focus on improving optimal mental health among these set of people.

Keywords: Mental healthcare, awareness, relationship status, educational level, COVID-19, Low-income earners, Western Uganda

\section{INTRODUCTION}

The world is now battling with a new type of virus that was identified by the Chinese Center for Disease Control and Prevention as the causative agent of pneumonia-like infection which present with cough and high fever first reported amongst patients in Wuhan city of 
China by the end of 2019 [1]; the virus, initially named 2019 nCoV by the World Health Organization (WHO) was later renamed severe acute respiratory syndrome coronavirus-2 (SARS-CoV-2) by the International Committee on Taxonomy of Viruses [2]. In mid-February 2020, WHO announced an epidemic disease caused by SARS-CoV-2 as coronavirus disease 2019 (COVID-19). While respiratory distress syndrome was initially reported as the primary pathology in patients with COVID-19, several systems have since been implicated including the nervous system [3]. As at $6^{\text {th }}$ of August 2020, over 18.6 million confirmed cases and 702642 death were reported globally. In Africa, 848053 confirmed cases and 15252 deaths were reported in WHO corona disease situation report [4].

While the extent of the damage caused by the COVID-19 pandemic in Africa remains unknown, its impact on the socioeconomic and mental health status is palpable. As for other coronaviruses, mental health symptoms (anxiety, depression and peritraumatic stress) have been reported during this COVID-19 crisis [5]. The pandemic impacts on the physical human health, social and political patterns a positive feedback that characteristically and indirectly inhibit the social, economic and political wellbeing of communities, societies and nations. As the pandemic progresses, it is likely that mental health burden will increase as measures taken by the African governments to mitigate the spread of the SARS-COV-2 virus, such as shelter-in-place order, banning public gatherings, closure of schools and social distancing have a great impact on the lives of low income earners, earning $\$ 1,025$ or less per month [6]. Strategies put in place to limit the spread of COVID-19 expose low income earners to experience situations that could be a predisposing factor to poor mental health outcomes, such as isolation and job loss [7].

Public health emergencies can have psychological effects on college students, expressed as anxiety, fear, and worry; economic effects, and effects on daily life, as well as delays in academic activities, have been positively associated with anxiety and social support was negatively correlated with the level of anxiety [8]. Not only is education affected within this shelter-in-place and physical isolation, intimate relationship and effective relationships among individuals are also affected.

The pandemic combined with social distancing is creating very stressful situations, and a fairly high number of people are exhibiting mental health symptoms such as fear, irritability, depression or anxiety; unavoidable in the face of a perceived threat. he fear of 
contracting the virus, negatively influences a rather well functioning romantic relationships as individuals have been recorded to have increased aggression, panic and hostility towards their partners. Previous studies have found a strong association between separation or divorce and an increased risk of depression, nevertheless, it is also asserted that marital relationships have noticeable beneficial effects on the mental health of couples $[\mathbf{9 , 1 0 ]}$. Effective communication, support and responsiveness are some factors that can help a relationship thrive during times of crisis and as well as enable partners maintain a stable mental health status. In a study by Steele et al. [11], it was observed that there is an association between educational status and anxiety and or the use of mental health services. In that study, individuals with higher education accessed mental health services more readily than those less likely to be recognised as educated. Similarly, a cross-national investigation of 12-month and lifetime prevalence of mental disorders were investigated among college students. The results showed that a significant number of the population studied have mental disorders which were substance abuse, anxiety disorders, mood disorders and behavioral disorders. In this case, anxiety disorders were the most prevalent among the college students [12]. In another study, students in higher education had a tendency not to complete their tertiary education as a result of mental health challenges; poor mental health was significantly associated with school dropouts among students [13].

Mental healthcare awareness has attracted widespread attention over the years. Higher levels of education are associated with increased access to a wide range of information, higher employment accessibility, improves health and promotes inter-personal and intra-personal well-being [14]. Lopez et al. [15] stated that, individuals who have attained some level of college education have a significantly higher knowledge of mental healthcare than their counterparts with low levels of education.

This study aimed to determine the effect of education level and relationship status on levels of awareness of mental healthcare and mental status among low income earners in Western Uganda. This data would be of value in guiding policy makers to evolve strategies focused at social transformation guided by effective mental health education and supporting low income earners towards resilience during public health emergencies such as the COVID-19 pandemic. 


\section{METHODOLOGY}

\section{Study site and design}

The study was a cross-sectional descriptive study, aimed at investigating the implications of education level and relationship status on level of awareness on mental healthcare and mental health state among low income earners in Western Uganda. The present study deployed simple random sampling technique.

\section{Study population}

Low income earners between 18 and 65 years of age living and or working within Ishaka municipality in Bushenyi district of Western Uganda who gave their consent to be part of the study were recruited. Those outside the age required for the study and those who had not consented to participate in the study were excluded from the study. Low income earners were individuals who depend on day to day business activities for their livelihood such as "boda" riders (cyclist), mobile money stand attendants, traders and the like.

\section{Data collection method and tool}

A closed-ended questionnaire was used to collect data from the respondents who met the inclusion criteria for the study. The questionnaire had questions covering five different areas of the study; sociodemographic (age, educational status and relationship status), awareness on mental healthcare, anxiety, anger and depression. Awareness of mental health care was assessed using simple questions. Anxiety was assessed using a modified generalized anxiety disorder (GAD-7) item tool. Anger was assessed using a modified Spielberger's State-Trait Anger Expression Inventory-2 (STAXI-2). Depression was assessed using a modified Beck Depression Inventory (BDI). Responses from the different components of the questionnaire were assigned scores. A Google format of the questionnaire was used to minimize physical contact and also maintain social distancing according to the guidelines by WHO and

Ministry of Health in Uganda. Respondents who could not understand the questions clearly had the questions interpreted in local 
language for them by team members who can communicate effectively in the local language. In the end 280 potential respondents were approached, out of whom 27 of them declined. Therefore, the sample size is 253.

\section{Data management and organization}

115 The data obtained from the survey was entered into Microsoft excel (2016) and scores were assigned to each option as follows: Mental 116 Health Care Awareness (Q5 - Q10): Numerical values - Mental Health Awareness [Correct response = 1, Incorrect response = 0]. 117 Modified GAD Assessment of Anxiety $(\mathrm{Q} 11-\mathrm{Q} 16)$ : Numerical values - Multiple response $[$ For each option selected $=1$, indifferent $=$ 118 0]. Modified STAXI-2 Assessment for Anger $(\mathrm{Q} 17-\mathrm{Q} 23)$ : Numerical values - Multiple response [For each option selected = 1, 119 indifferent = 0]. Modified BDI Assessment for Depression $($ Q24 - Q30): Numerical values - Single graded response [Highest grade of 1203 , indifferent $=0$ ]. However, the data collected were assessed for completeness and responses failing to meet the $75 \%$ cut-off (on all 121 valid questions) were excluded (Figure 1).

122 For questions on awareness level, every correct response was assigned (1) and incorrect response was assigned (0). The scores of the 123 multiple options for the modified GAD, and STAXI-2 were obtained by assigning one (1) mark per response. While BDI had four (4) 124 options graded as 3, 2, 1 and 0 (for indifferent). For specific graded questions (Yes, sometimes, or No), scores; 2, 1, 0 were assigned and all questions in this form were cumulated (per row) and the averages were obtained by summing all scores (qt) and dividing the number of questions ( $\mathrm{n}$ ) for each section. This scoring and grading system allows for both linear and uni/multivariate logistic regression analysis. On the other hand, the obtained scores for each individual in the different segment was then converted to percentage, so as to get the mean percentage score for awareness, anxiety, anger and depression. 


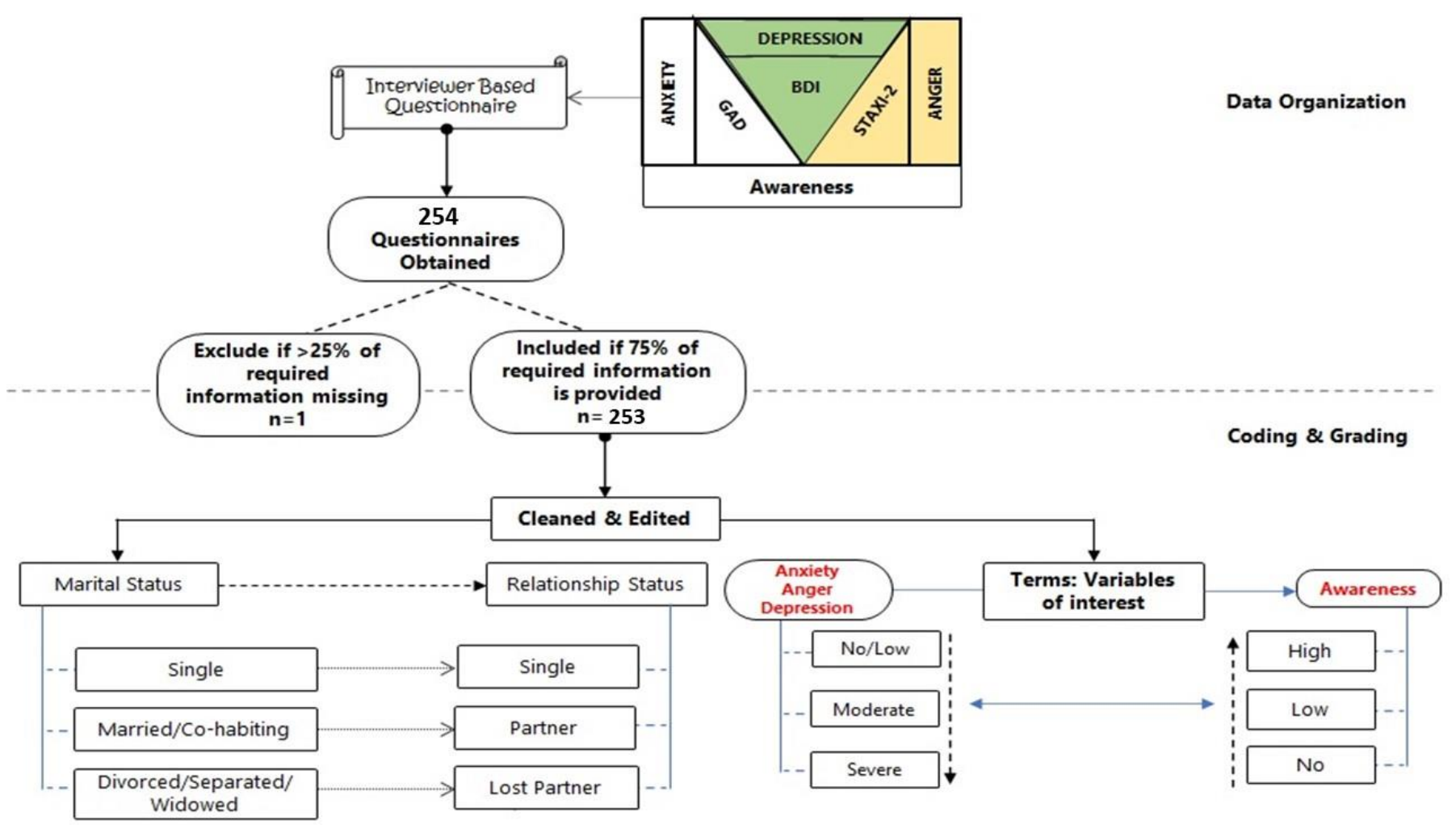

132 Figure 1: Data Management (Organization, coding, \& grading)

\section{Data Analysis}

134 The data was transferred to Graphpad Prism version 6 and Minitab® 18.1 (Minitab, Inc. 2017) for analysis. The relationship between 135 educational status and relationship status with awareness, anxiety, anger and depression level we examined using Spearman Rho 136 correlation, then all significant correlates were regressed using system-assisted regression model. All analyses were performed at 95\% 137 confidence level and p-values less than 0.05 were taken to be significant. 
140 The majority of our respondents were male 150/253 (59.3\%) who had attained secondary education level 104/253 (41.1\%) and were single 137/253 (54.2\%). Respondents with tertiary level education and those with partners exhibited higher scores (71.7\% and $70.28 \%$ respectively) for awareness. Respondents with primary education level and single individuals had a higher mean score (46.98\% and $43.72 \%$ respectively) for anxiety. Respondents with primary education level and those leaving with partners had higher mean score (56.44\% and 56.21\% respectively) for anger. Respondents with no formal education and those who had lost partners had higher mean score $(32.11 \%$ and $30.50 \%$ respectively) for depression (Figure 1$)$.

Educational status was observed to influence depression as age increases. The individuals without formal educational and primary education were observed to have a negative correlation ( $\mathrm{r} 2=47.4$ and $6.4 \%$ respectively) with awareness on mental healthcare, and the no formal group had positive correlation with anger and depression ( $\mathrm{r} 2=1.9$ and $0.3 \%$ respectively), while other groups were negatively correlated ( $\mathrm{r} 2=10.1 \%$ ) (Table 2; Figure 2). In comparing the age associated chances across the relationship status group, it was observed that the lost-partner group had older individuals and were more aware, but also more depressed as age increased when compared to the single groups which has a negative correlation $(\mathrm{r} 2=1.9 \%)$ with awareness on mental healthcare, positive correlation $(\mathrm{r} 2=3.9 \%)$ with anxiety and negative correlation with anger and depression ( $\mathrm{r} 2=0.8$ and $0.3 \%$ respectively) (Table 2; Figure 3).

\section{DISCUSSION}

Awareness on mental healthcare is intrinsically linked with increased utilization of mental healthcare services. This study showed an increased awareness on mental healthcare with increase in age among the separated (divorced/widowed) and those living with a partner (Married) but decreased in singles group (never married) (Fig. 3). The increase in awareness on mental healthcare is likely due to the fact that being married (either previously or currently) is beneficial to mental health and influences awareness on mental health care [16][17][18], as such may have influenced the level of awareness on mental health care in these groups compared to the single individuals group. There was increase in awareness on mental healthcare with increase in age among people who had attained tertiary and secondary 
level of education compared to those who had primary and no formal education in the present study (Fig. 2). This means that educational level significantly contributed to mental healthcare awareness in this study. Primary level of education alone did not provide the needed exposure to respondents in terms of knowledge on certain aspects of life such as mental health care, thereby making it no different from respondents who had no formal education in terms of awareness on mental healthcare.

Our findings show that anxiety score increased among low income earners who were single individuals compared to those with partners (married) and those that had lost their partners (separated/widowed) (Fig. 1). Single people experienced more anxiety during the COVID19 lockdown in Western Uganda compared to those who are living with a partner or had once been in a marital relationship. Intimate relationships tend to provide an avenue for people to express and manage personal distress, which may help alleviate anxiety [19]. Marriage is associated with psychological benefits that make the partners happier and healthier [20]]21][22]. Zaider and colleagues reported that in marital relationships the wives were more vulnerable to anxiety than husbands and those wives often perceived that their husbands were the ones who alleviated or appeased their anxiety [19]. A study on "Singlism" observed that participants recruited in the study described married people as happy, fulfilled, stable, reliable, kind, giving and loving, while on the other hand, single people were described as unsuccessful and ugly [23], thus portraying the pressures and expectations on single people which may contribute to anxiety over their relationship status, especially in a lockdown. In addition, people who are single have been reported to negatively evaluate other singles as being more at risk and unstable [24]. Anxiety scores were decreased in those who had formal (primary, secondary and tertiary) and no formal education groups (Fig. 1 \& Fig. 2); having either educational or no educational exposure did not influence anxiety among low income earners in Western Uganda during the lockdown.

Anger score increased with age in respondents who had no formal education and primary education level when compared to those who had secondary and tertiary level of education. This trend is in line with the study done by Boylan and Ryff [25], who reported that educational status was linked to higher anger-control. The lack of formal education and low education may have been the reasons the no formal education and primary education level groups experienced an increase in anger score during the COVID-19 lockdown in Western Uganda. We also observed that the group without formal education had an increased depression score which increased with 
age while the primary, secondary and tertiary education groups had decreased depression scores. This is supported by Bjelland et al. [26], who reported that low education levels were significantly associated with depression. Another study by Ross and Mirowsky [27], stated that education is a resource that is an integral part of a person, rather than being an external part of a person. Mirowsky and Ross [27], postulated that education precedes and actually influences social economic status indicators such as occupation and income.

This study also showed an increase in depression score with an increase in age in lost partner group when compared to partner and single groups (Fig. 1 \& Fig. 3). This is in line with previous studies that reported separation or divorce to be associated with increased risk of depression, although, there are inconsistencies as to whether the increase is higher among women [28, 29, 9]. Being previously married (lost partner/separated) was associated with increased risk of depressive disorders; thereby supporting the fact that getting married was more protective against depression and alcohol use [30], and may be the reasons behind the increase in the depression score in separated groups when compared to partner and singles group in our study. An initial increase in anger score was observed among the separated groups but later decreased with age. This may be a confirmation to the fact that the separated group experienced more depression because anger is one of the ways most people express depression [31].

\section{CONCLUSION}

This study aimed to determine the effect of education level and relationship status on levels of awareness of mental healthcare and mental status among low income earners in Western Uganda. It is evident that education and relationship status influence awareness on mental healthcare and mental health state among low income earners in Western Uganda. This data would be of value in guiding policy makers to evolve strategies focused at social transformation guided by effective mental health education and supporting low income earners towards resilience during public health emergencies such as the COVID-19 pandemic. We advocate for new approaches in mental healthcare awareness programs equipped with robust strategy, which will focus on improving optimal mental health among this group of individuals. 


\section{ABBREVIATION}

204 nCoV: Novel coronavirus

205 SARS-CoV-2: Severe acute respiratory syndrome coronavirus-2

206 WHO: World Health Organization

207 BDI: Beck Depression Inventory

208 GAD: Generalized anxiety disorder

209 STAXI-2: Spielberger's State-Trait Anger Expression Inventory-2

\section{DECLARATIONS}

\section{Ethical approval and consent to participate}

212 Expediated ethical approval from Kampala International Ethical Review Board was acquired and registered as Nr.UG-REC-023/201914.

213 Introductory letter was also obtained from community head to facilitate community entry especially with the Covid 19 lockdown.

214 Informal consent was acquired from each participant before data collection.

\section{Consent for publication}

216 Not required

\section{Data availability}

218 Data files can be accessed at https://figshare.com/s/bc18d5417d965c15a283

\section{Competing interests}

220 The authors declare no conflicts of interest. 


\section{Funding}

222 Not applicable

\section{Author contributions}

224 AML, KIK, IMU conceptualized the study; AML, KIK, IMU, VAB, SCW designed the study; IJ, RS, AMA, SDT, NEM, AA, JOA, 225 OS, FS, JTA, KM, RH, SK, HP, TP collected the data; EOA, VAB, IJ, conducted statistical analysis, , EOA, VAB, IJ, RS, AMA, SDT, 226 NEM, AA, JOA, OS, FS, JTA, KM, RH, HY, SK, HP, TP, SCW, conducted data interpretation. AML, KIK, IMU, NEM drafted the 227 initial manuscript while AML, KIK, IMU, EOA, VAB, IJ, RS, AMA, SDT, NEM, AA, JOA, OS, FS, JTA, KM, RH, HY, SK, HP, TP,

228 SCW reviewed it for intellectual content. All authors approved the final version for publication and remain in agreement to ensure that 229 questions related to the integrity of any part of the work are resolved.

\section{Supplementary file}

231 Supplementary file 1 . The questionnaire.

\section{Acknowledgments}

233 Authors are grateful to colleagues who offered positive criticism and are not on the paper.

\section{REFERENCES}

1. Chen N, Zhou, M, Dong X, Qu J, Gong F, Han Y, Zhang L. Epidemiological and clinical characteristics of 99 cases of 2019 novel coronavirus pneumonia in Wuhan, China: a descriptive study. 2020; The Lancet. https://doi.org/10.1016/S01406736(20)30211-7

2. World Health Organization 'Naming the coronavirus disease (COVID-19) and the virus that causes it', World Health 
Organization. 2020;

3. Asadi-Pooya AA. \& Simani, L. Central nervous system manifestations of COVID-19: A systematic review. Journal of the Neurological Sciences. 2020; https://doi.org/10.1016/j.jns.2020.116832

4. World Health Organization. Coronavirus (COVID-19) Situation Report - 199. Published by World Health Organization on $6^{\text {th }}$ August 2020 and retrieved on $10^{\text {th }}$ of August 2020.

5. Chen Y, Zhou H, Zhou, Y \& Zhou F. Prevalence of self-reported depression and anxiety among pediatric medical staff members during the COVID-19 outbreak in Guiyang, China. Psychiatry Research.2020; https://doi.org/10.1016/j.psychres.2020.113005

6. George M. Uganda has insufficient supply of houses for low income earners - regional think tank. Published by eagles online on November 4, 2019 and retrieved on $6^{\text {th }}$ of August 2020. https://eagle.co.ug/2019/11/04/uganda-has-insufficient-supply-ofhouses-for-low-income-earners-regional-think-tank.html

7. Blustein DL, Duffy R, Ferreira J A, Cohen-Scali V, Cinamon RG \& Allan BA. Unemployment in the time of COVID-19: A research agenda. Journal of Vocational Behavior. 2020; https://doi.org/10.1016/j.jvb.2020.103436

8. Cao W, Fang Z, Hou G, Han M, Xu X, Dong J, \& Zheng J. The psychological impact of the COVID-19 epidemic on college students in China. Psychiatry Research. 2020; https://doi.org/10.1016/j.psychres.2020.112934

9. Hope S, Rodgers B, Power C. Marital status transitions and psychological distress: longitudinal evidence from a national population sample. Psychological Medicine 1999; 29, 381-389

10. Scott KM, Wells JE, Angermeyer M, Brugha TS, Bromet E, Demyttenaere K, de Girolamo G, Gureje O, Haro JM, Jin R, NasserKaram A, Kovess V, Lara C, Levinson D, Ormel J, Posada-Villa J, Sampson N, Takeshima T, Zhang M and Kessler RC. Gender and the relationship between marital status and first onset of mood, anxiety and substance use disorders. Psychological Medicine. 2009.

11. Steele LS, Dewa CS, Lin E, \& Lee KL. Education level, income level and mental health services use in Canada: associations and policy implications. Healthcare policy = Politiques de sante, 2007; 3(1), 96-106. 
12. Auerbach, RP, Alonso J, Axinn WG, Cuijpers P, Ebert DD, Green JG, Caldas-de- JM.. HHS Public Access. 46(14), 29552970. 2017; https://doi.org/10.1017/S0033291716001665.Mental

13. Hjorth CF, Bilgrav L, Frandsen LS, Overgaard C, Torp-pedersen C, Nielsen B, \& Bøggild H. Mental health and school dropout across educational levels and genders : a 4 . 8-year follow-up study. BMC Public Health, 2016; 1-12. https://doi.org/10.1186/s12889-016-3622-8

14. Anna Z and Elizabeth ML. The Relationship Between Education and Health: Reducing Disparities Through a Contextual Approach._Annual Review of Public Health. 2018; 39:1, 273-289. https://doi.org/10.1146/annurev-publhealth-031816-044628

15. Lopez V, Sanchez K, Killian MO. et al. Depression screening and education: an examination of mental health literacy and stigma in a sample of Hispanic women. BMC Public Health 18, 646 (2018). https://doi.org/10.1186/s12889-018-5516-4

16. Gove W and Tudor J. F (1973). Adult sex roles and mental illness. American Journal of Sociology 78, 812-835

17. Wu X and Demaris A. Gender and Marital Status Differences in Depression: The effects of chronic strains. Sex Roles 34, 299320. 1996

18. Kiecolt-Glaser JK and Newton TL. Marriage and Health: His and Hers. Psychological bulletin. 2001; 127, 472-503

19. Zaider TI, Heimberg RG and Iida M. Anxiety Disorders and Intimate Relationships: A Study of Daily Processes in Couples. $J$ Abnorm Psychol 119(1): 163-173. 2010; doi: $10.1037 / \mathrm{a} 0018473$

20. Adamczyk K and Segrin C. Perceived social support and mental health among single vs partnered polish young adults. Current psychology: A journal for diverse perspectives on diverse psychological issues. 34, 82-96. 2005; https://doi.org/10.1007/s12144$\underline{014-9242-5}$

21. Pierewan AC and Tampubolon G. Happiness and health in Europe: A multivariate multilevel model. Applied Research Quality of Life. 10.237-252. 2015; https://doi.org/10.1007/s11482-014-9309-3

22. Ta VP, Gesselman AN, Perry BL, Fischer HE and Garcia JR. Stress of singlehood: Marital status, domain-specific stress and anxiety in a national U. S. sample. Journal of social science and clinical psychology 2017; 36, 461-485 
23. Depaulo BM and Morrris WL. The unrecognized stereotyping and discrimination. Current Directions in Psychological Science.2006; 15, 251-254

24. Conley TD and Collins BE. Gender, relationship status and stereotyping about sexual risk. Personality and social psychology bulletin 2002; 28, 1483-1494

25. Boylan JM and Ryff CD. Varieties of Anger and the Inverse Link between Education and Inflammation: Toward an Integrative Framework. Psychosom Med 2013; 75(6): 566-574

26. Bjelland I, Krokstad S, Mykletun A, Dahl AA., Tell GS, Tambs K. Does a higher educational level protect against anxiety and depression?. The HUNT study. Social Science and Medicine.2008; Doi.10.1016/j.socscimed.2007.12.019

27. Ross CE and Mirowsky J. Sex differences in the effect of education on depression: resource multiplication or resource substitution? Social Science and Medicine.2006; 63(5): 1400-1413

28. Mirowsky J and Ross CE. Education, Personal control, life-style and health. A human capital hypothesis. Research on Aging. 1998; 20-415-449

29. Marks NF and Lambert JD Marital status continuity and change among young and midlife adults: Longitudinal effects on psychological well being. Journal of family issues 1998; 19, 652-697

30. Horwitz AV, White HR, Howell-White S. Becoming married and mental health: a longitudinal study of a cohort of young adults. Journal of Marriage and the Family. 1996;58, 895-907.

31. Timothy J Legg and Rachell Nall. What is the link between depression and anger? January 3, 2020; Available: https://www.medicalnewstoday.com/articles/327408 accessed on 7/7/2020 
309 Table 1: Demographics features of the study population

\begin{tabular}{llcc}
\hline Characteristics & Categories & Frequency (\%) & 95\% CI \\
\hline Sex & Male & $150(59.3)$ & 53.1 to 65.2 \\
& Female & $103(40.7)$ & 34.8 to 46.9 \\
Education level & No formal education & $9(3.6)$ & \\
& Primary & $41(16.2)$ & 1.8 to 6.4 \\
& Secondary & $104(41.1)$ & 35.2 to 47.3 \\
& Tertiary & $99(39.1)$ & 33.3 to 45.3 \\
Relationship status & Lost partner & $4(1.6)$ & \\
& Partners & $112(44.2)$ & 38.5 to 3.8 \\
& Single & $137(54.2)$ & 48.0 to 60.2 \\
\hline
\end{tabular}

310 Sample size $=253$

311 CI: Confidence interval, \%: Percentage

312 

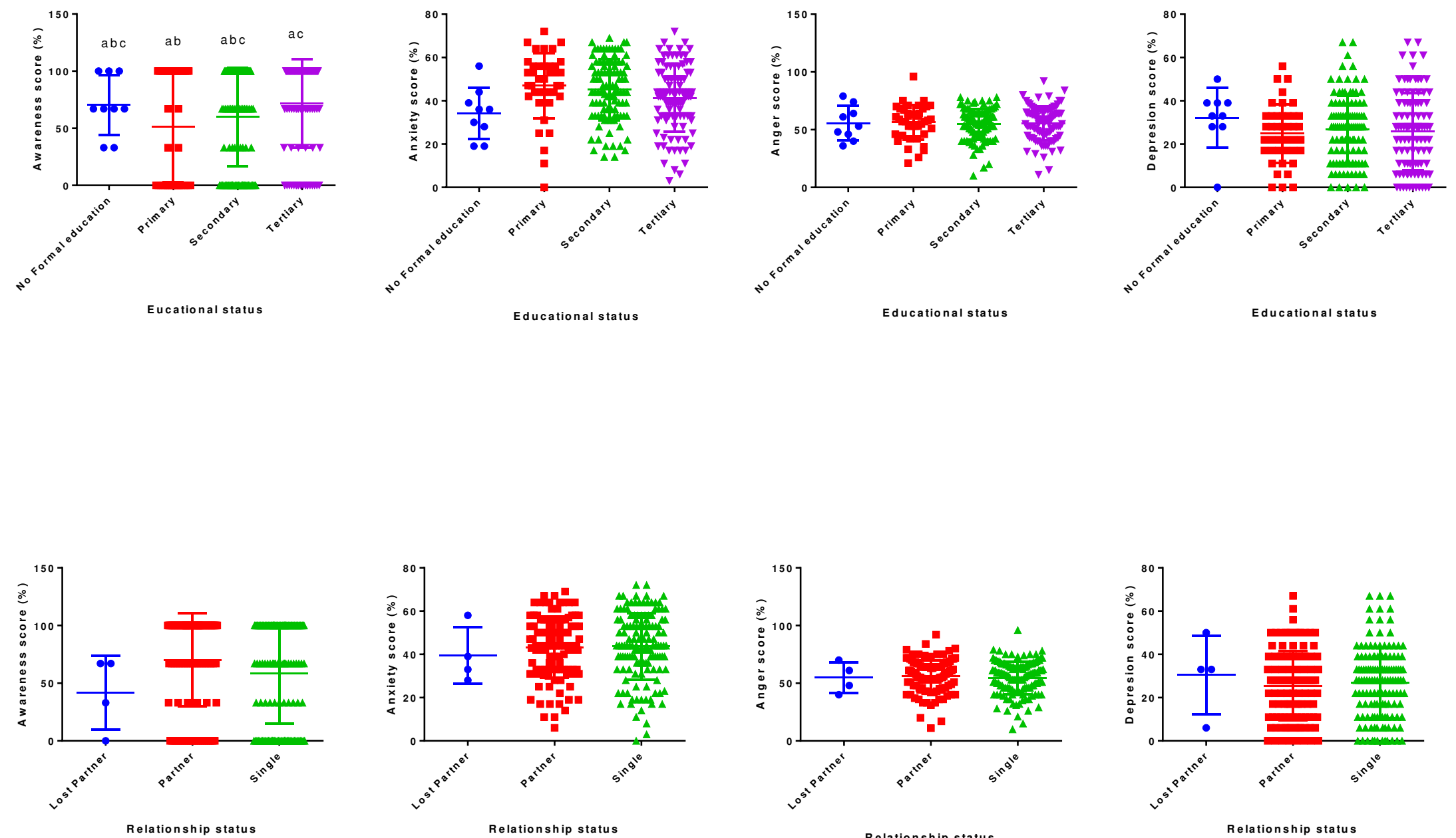

Figure 1: Score for Awareness, anxiety, anger and depression among various Education and relationship status 
Table 2: Relationship (accuracy $\left[\mathbf{R}^{2}\right]$ and direction) of age with awareness, anxiety, anger and depression; stratified by sociodemographic characteristics

\begin{tabular}{llcccc}
\hline \multirow{2}{*}{ Variables } & & \multicolumn{3}{c}{ Age (accuracy: \% [relationship]) } \\
& & Awareness & Anxiety & Anger & Depression \\
\hline \multirow{2}{*}{ Educational Status } & No formal & $47.4 \%(-\mathrm{ve})$ & $10.1 \%(-\mathrm{ve})$ & $1.9 \%(+\mathrm{ve})$ & $0.3 \%(+\mathrm{ve})$ \\
& Primary & $6.4 \%(-\mathrm{ve})$ & $1.3 \%(+\mathrm{ve})$ & $2.5 \%(-\mathrm{ve})$ & $3.0 \%(-\mathrm{ve})$ \\
& Secondary & $4.5 \%(+\mathrm{ve})$ & $0.0 \%(+\mathrm{ve})$ & $1.6 \%(-\mathrm{ve})$ & $0.6 \%(-\mathrm{ve})$ \\
& Tertiary & $0.1 \%(+\mathrm{ve})$ & $0.0 \%(+\mathrm{ve})$ & $0.2 \%(-\mathrm{ve})$ & $6.4 \%(-\mathrm{ve})$ \\
& Lost-Partner & $47.0 \%(+\mathrm{ve})$ & $87.6 \%(-\mathrm{ve})$ & $48.9 \%(-\mathrm{ve})$ & $16.4 \%(+\mathrm{ve})$ \\
& Partner & $3.7 \%(+\mathrm{ve})$ & $0.3 \%(-\mathrm{ve})$ & $1.5 \%(-\mathrm{ve})$ & $8.1 \%(-\mathrm{ve})$ \\
& Single & $1.9 \%(-\mathrm{ve})$ & $3.9 \%(+\mathrm{ve})$ & $0.8 \%(-\mathrm{ve})$ & $0.3 \%(-\mathrm{ve})$ \\
\hline
\end{tabular}

Sample size $=253$

$\%$ : Percentage, +ve: Positive, -ve: Negative

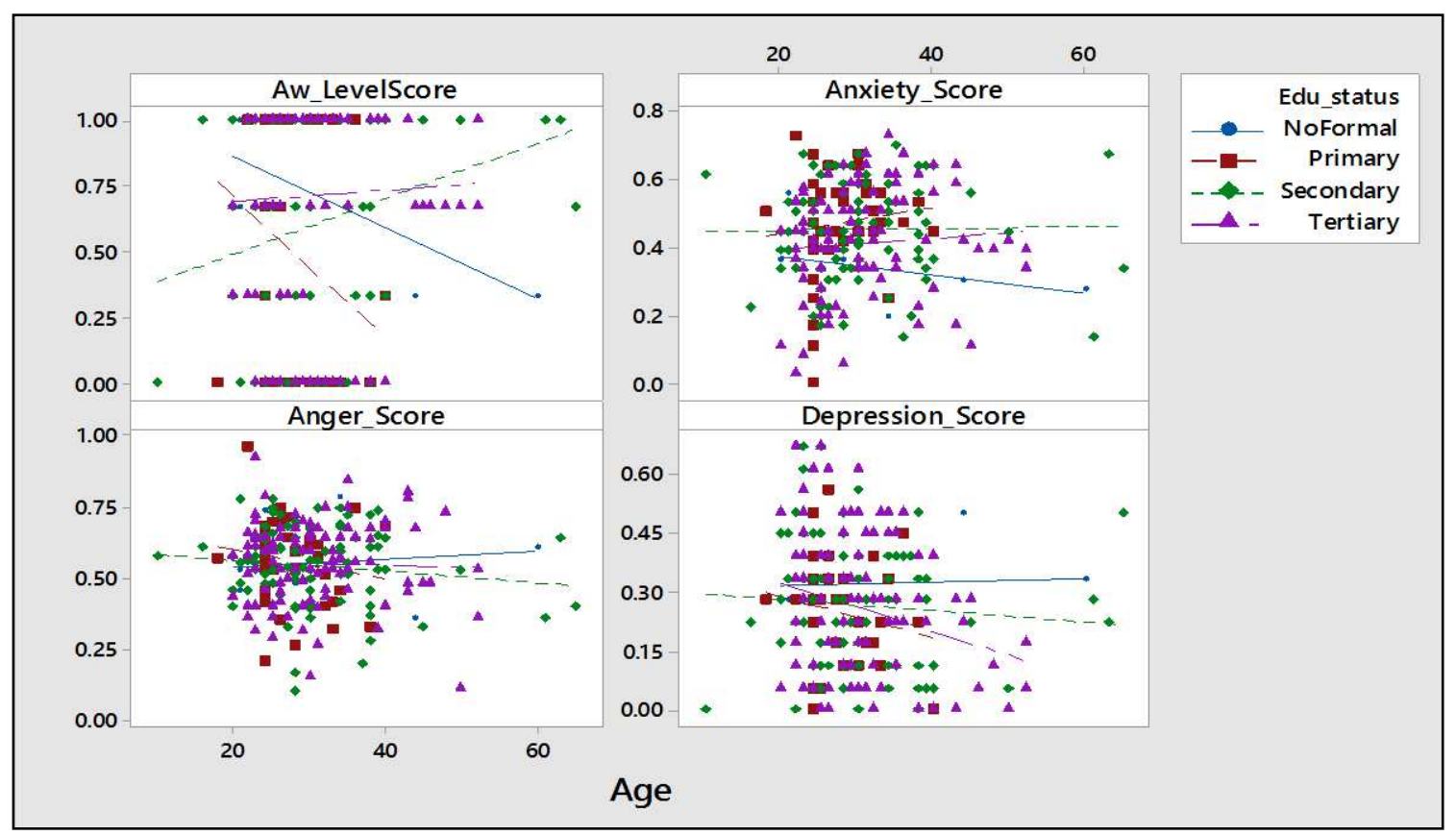

Figure 2: Scatter plot of Awareness, Anxiety, Anger and Depression vs Educational Status

AW: awareness, Sample size $=253$ 


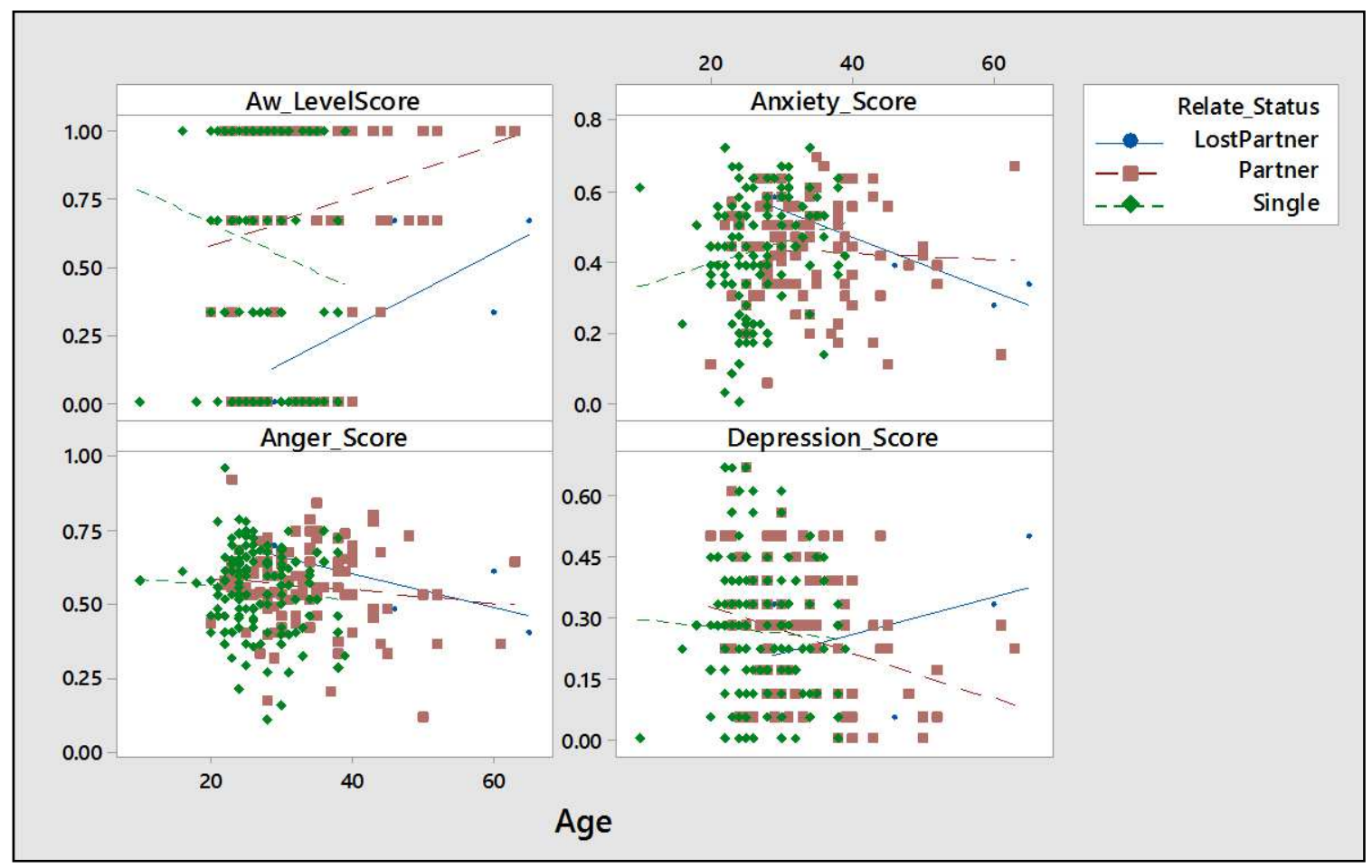

Figure 3: Scatter plot of Awareness, Anxiety, Anger and Depression vs Relationship Status

AW: awareness, Sample size $=253$ 


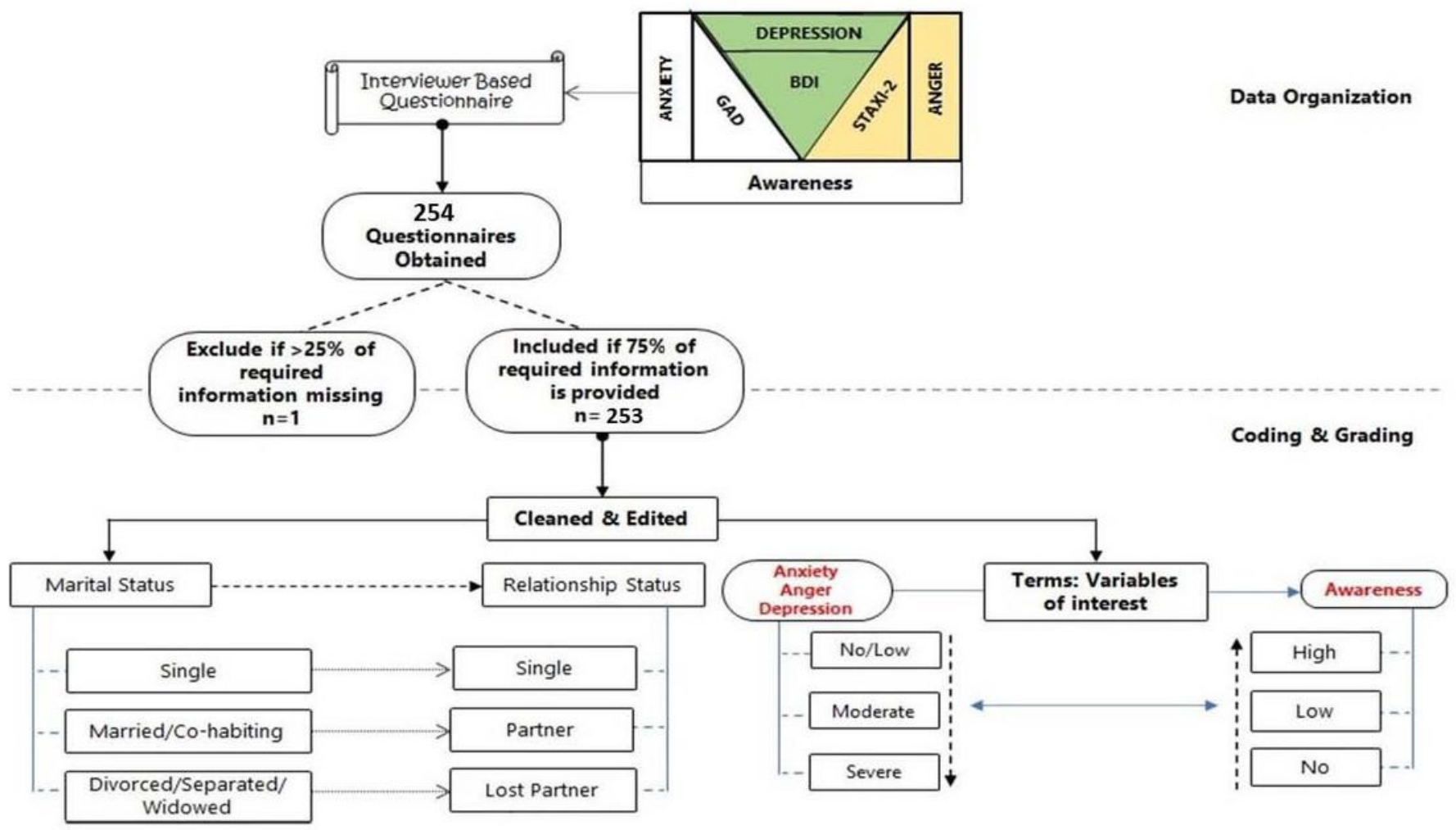

Figure 1

Data Management (Organization, coding, \& grading) 


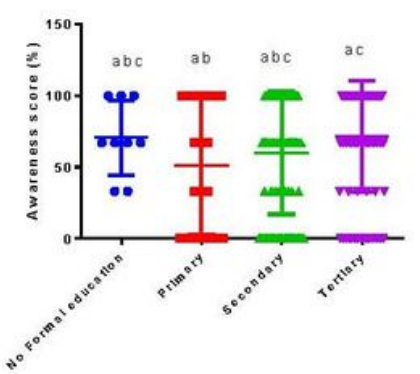

Eucational status

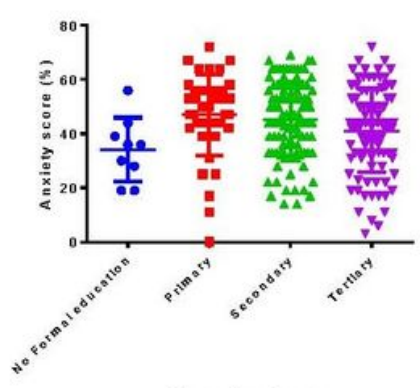

Educational status

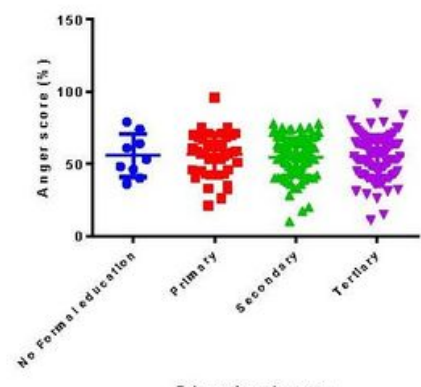

Educational s tatus

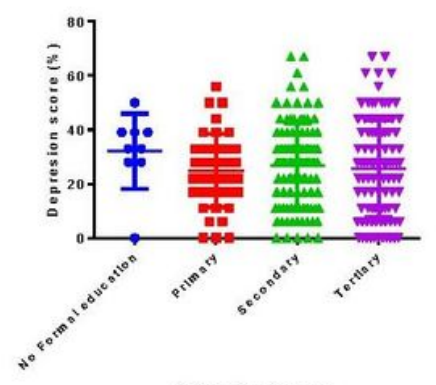

Educational status

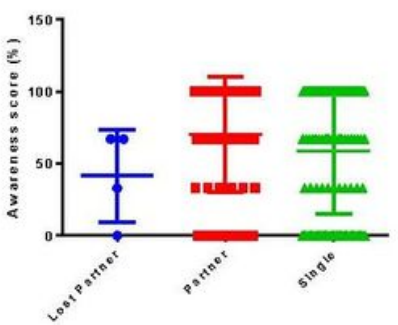

Relationship status

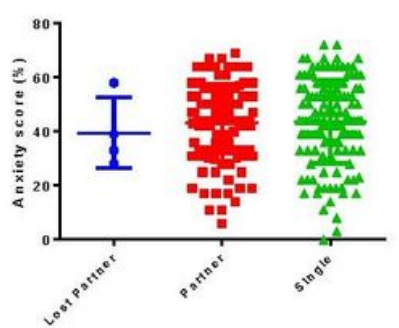

Relationship status

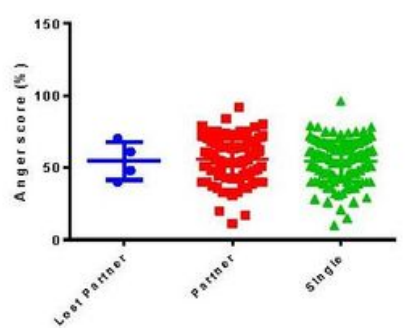

Relationship status

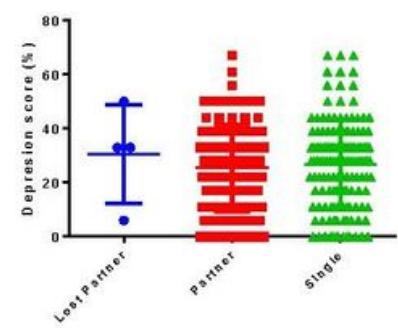

Relationship status

Figure 2

Score for Awareness, anxiety, anger and depression among various Education and relationship status

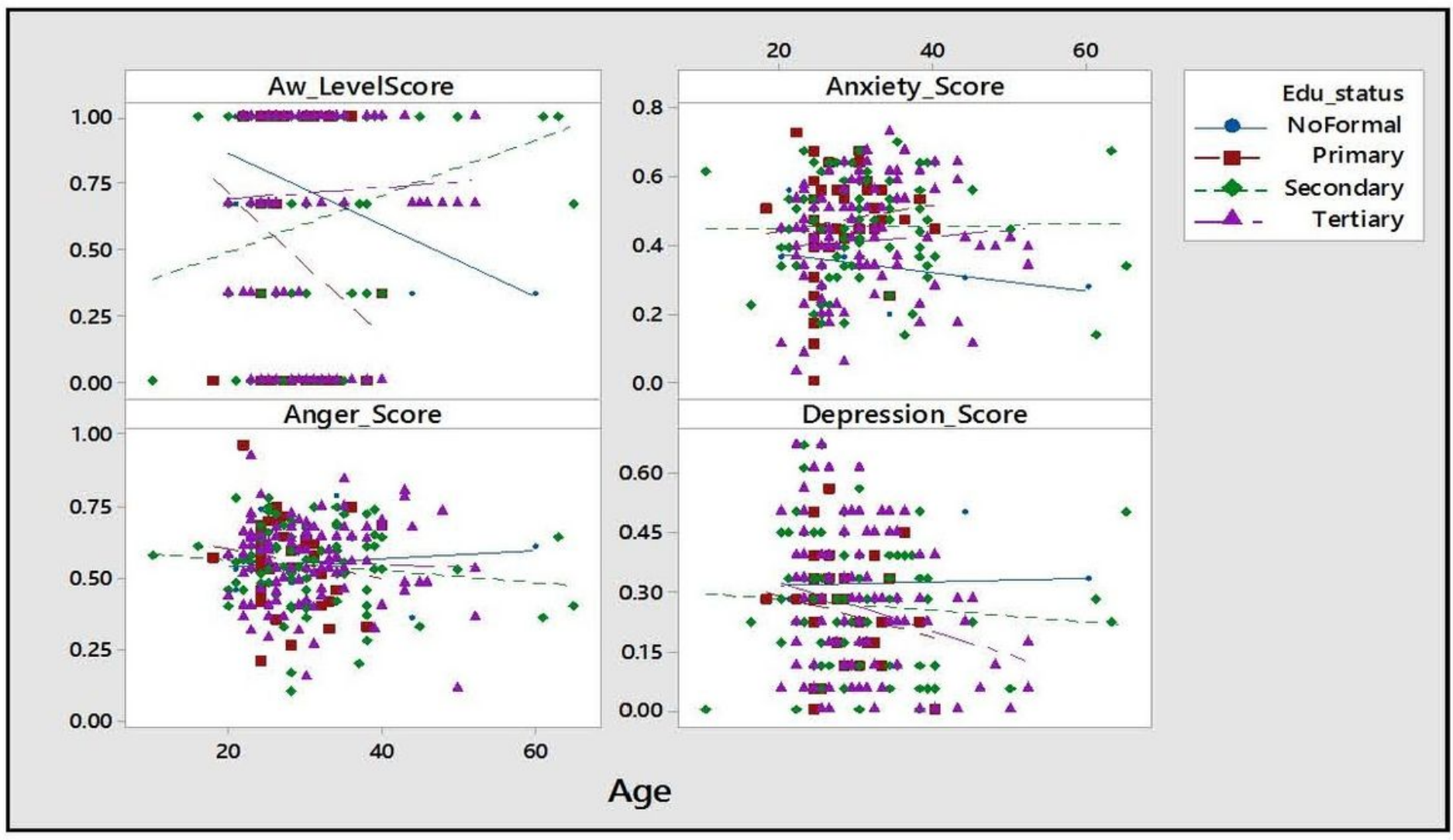




\section{Figure 3}

Scatter plot of Awareness, Anxiety, Anger and Depression vs Educational Status AW: awareness, Sample size $=253$

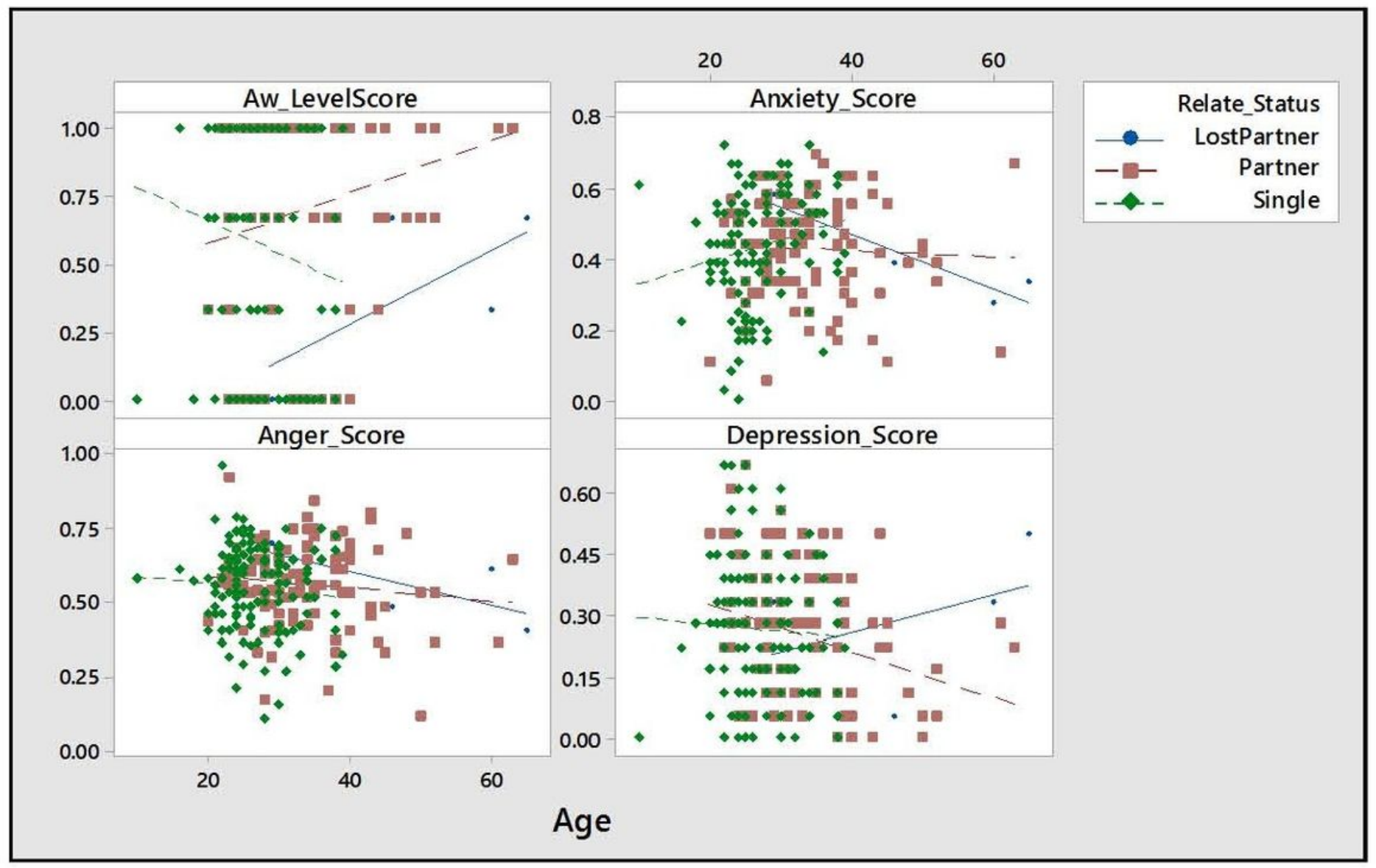

\section{Figure 4}

Scatter plot of Awareness, Anxiety, Anger and Depression vs Relationship Status AW: awareness, Sample size $=253$

\section{Supplementary Files}

This is a list of supplementary files associated with this preprint. Click to download.

- AnnQuestionaireMentalhealth.docx

- AnnQuestionaireMentalhealth.docx 